\title{
Jet propagation and medium excitation in a quark-gluon plasma ${ }^{1}$
}

\author{
Tan $\mathrm{Luo}^{\mathrm{a}}$, Yayun $\mathrm{He}^{\mathrm{a}}$, Xin-Nian Wang ${ }^{\mathrm{a}, \mathrm{b}}$, Yan Zhu ${ }^{\mathrm{c}}$ \\ ${ }^{a}$ Key Laboratory of Quark and Lepton Physics (MOE) and Institute of Particle Physics, Central China Normal University, Wuhan 430079, China \\ ${ }^{b}$ Nuclear Science Division Mailstop 70R0319, Lawrence Berkeley National Laboratory, Berkeley, California 94740, USA \\ ${ }^{c}$ Departamento de Física de Partículas and IGFAE, Universidade de Santiago de Compostela, E-15706 Santiago de Compostela, Galicia, Spain
}

\begin{abstract}
We implement the complete set of elastic $2 \rightarrow 2$ parton scattering processes in the Linearized Boltzmann Transport (LBT) model to study the parton propagation inside a hot quark-gluon plasma. We calculate and compare the elastic energy loss and the transverse momentum distribution of quarks and gluons. We further simulate a single jet propagation and the induced medium excitation within a static quark-gluon plasma to study how the jet energy and profiles are modified by the jet-medium interaction and in particular the jet-induced wake. Effects of the recoiled thermal partons and the jet-induced wake on the jet energy loss and profiles are studied in detail.
\end{abstract}

Keywords: Jet quenching, jet transport, parton energy loss, quark-gluon plasma

Introduction. When a hard parton produced at the very beginning of a heavy-ion collision propagates through the quark-gluon plasma (QGP), the recoiled partons and radiated gluons due to jet-medium interaction will lead to parton energy loss and jet quenching [1] as discovered in heavy-ion collisions at the the Relativistic Heavy-ion Collider (RHIC) [2,3] and observed again at the Large Hadron Collider (LHC) [4, 5]. Although induced gluon radiation is the major source of the parton energy loss, collisional energy loss due to elastic $2 \rightarrow 2$ scattering processes may also be important to describe the observed results at RHIC and LHC as indicated by recent studies [6, 7, 8,9]. The jet-medium interaction will also lead to $p_{T}$ broadening, which describes the accumulation of deflection of the high energy partons via multiple collisions. Besides the transverse momentum transfer and the energy loss, multiple interaction with the thermal partons from the medium may also change the flavor and species of the leading parton in the annihilation and creation processes. Therefore a complete set of elastic processes in QCD has been implemented in our Linearized Boltzmann Transport (LBT) Monte Carlo model.

As shown in the previous work [12] within the LBT model [15] with the approximation of small angle scattering, the energy loss of reconstructed jets is found to be reduced significantly after the inclusion of the recoiled thermal partons. This indicates that effects of the recoiled partons from the medium are not negligible in the full jet study, and the implementation of complete set of elastic scattering processes is needed to give a more accurate and detailed study of such effects. We will concentrate only on the elastic scattering processes in this study, and simulate the propagation of a single jet parton and jet-induced medium excitation in a static and uniform QGP. We use a modified version of the anti- $k_{t}$ algorithm in FASTJET [13] to reconstruct the leading jet and study the jet energy loss and structure modification in the hot medium.

In the simulations of interaction between leading, recoiled and thermal partons in our LBT model, when a parton 1 of type i travels through the QGP, it can scatter with a thermal parton 2 of type $\mathrm{j}$ (light quark, antiquark or gluon). The

${ }^{1}$ Talk presented by T. Luo at QM2014 
scattering rate for such process is $\Gamma_{i}=\sum_{j(k l)} \Gamma_{i j \rightarrow k l}$, where $\Gamma_{i j \rightarrow k l}$ is the scattering rate of the two partons via a specific channel [16]. Once we determine that there is a parton-medium scattering according to the Poisson distribution $P_{i}=1-e^{-\Gamma_{i} \Delta t}$ in a time step $\Delta t$, we use the single channel scattering rate as the weight to sample the every channel of this collision. We simulate each scattering process via the Boltzmann transport equation,

$$
p_{1} \cdot \partial f_{1}\left(p_{1}\right)=-\frac{1}{2} \int \frac{d^{3} p_{2}}{(2 \pi)^{3} 2 E_{2}} \int \frac{d^{3} p_{3}}{(2 \pi)^{3} 2 E_{3}} \int \frac{d^{3} p_{4}}{(2 \pi)^{3} 2 E_{4}}\left(f_{1} f_{2}-f_{3} f_{4}\right)\left|M_{12 \rightarrow 34}\right|^{2} S_{2}(s, t, u)(2 \pi)^{4} \delta^{4}\left(p_{1}+p_{2}-p_{3}-p_{4}\right),
$$

where $f_{i}=(2 \pi)^{3} \delta^{3}\left(\vec{p}-\vec{p}_{i}\right) \delta^{3}\left(\vec{x}-\vec{x}_{i}-\vec{v}_{i} t\right)(i=1,3)$ are the jet shower partons phase-space densities in the initial and final stage of the scattering. The thermal parton phase-space distributions $f_{i=2,4}\left(p_{i}\right)$ are Bose-Einstein distribution for gluons and Fermi-Dirac distribution for quarks and antiquarks in a thermal medium with the fluid velocity $u=(1, \vec{v}) / \sqrt{1-\vec{v}^{2}}$ and local temperature $T .\left|M_{12 \rightarrow 34}\right|^{2}$ are the matrix elements calculated with pQCD in terms of standard Mandelstam variables, which are constrained by a Lorentz-invariant regularization condition $S_{2}(s, t, u)=\theta\left(s \geq 2 \mu_{D}^{2}\right) \theta\left(-s+\mu_{D}^{2} \leq\right.$ $t \leq-\mu_{D}^{2}$ ) [16], where $\mu_{D}^{2}$ is the Debye screening mass squared. As we do not introduce a running strong coupling constant in this study, the strong coupling constant $\alpha_{\mathrm{s}}=g^{2} / 4 \pi$ is fixed at 0.3 throughout this paper.

We track both the jet shower partons $k\left(p_{3}\right)$ and the recoiled partons $l\left(p_{4}\right)$ after each scattering and let them scatter further in the medium. The initial thermal parton $j\left(p_{2}\right)$ in each scattering process is also transported according to the Boltzmann equation. It is denoted as a "negative" parton for its contribution has to be subtracted in the final results to take into account the back reaction in the Boltzmann equation. As a thermal parton moves from one phase space $j\left(p_{2}\right)$ to another phase space $l\left(p_{4}\right)$ during an elastic scattering process, the depletion of the initial phase space is described by the subtraction of the "negative" parton, which is the cause for the jet-induced wake that follows a propagating jet [15]. Since these partons from the wake are part of the jet-induced medium excitation therefore they should be taken into account in the detailed study of jet transport.

Results. We start with the elastic energy loss and transverse momentum distribution of the leading parton within the LBT model. In Fig. 1 we show the path length (propagation time) dependence of the elastic energy loss per unit
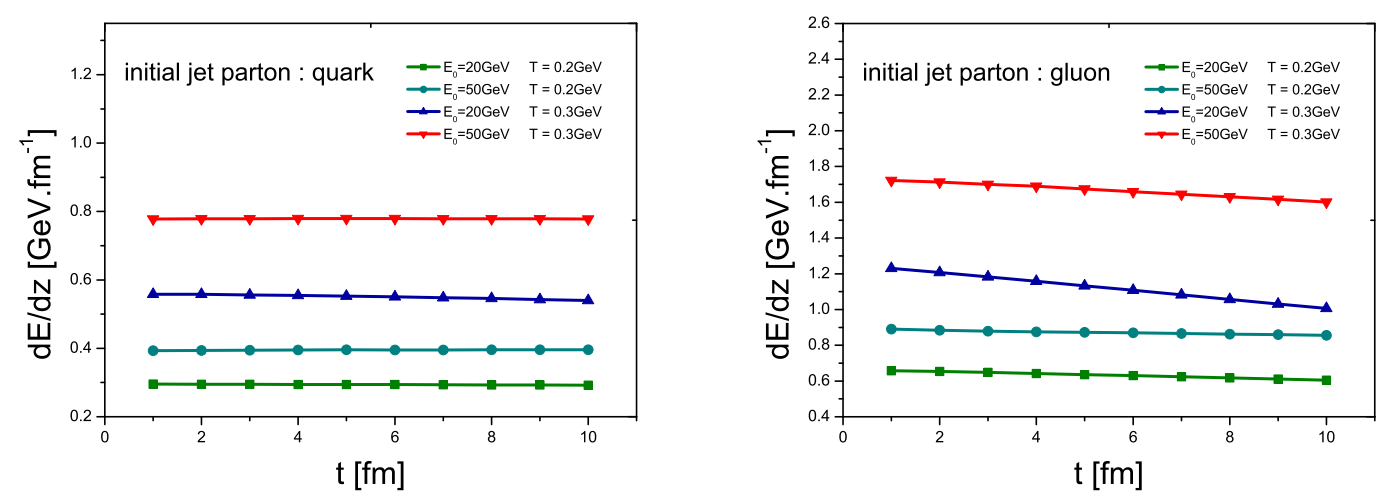

Figure 1. The path length dependence of the elastic energy loss per unit path length $d E / d z$ of a initial quark jet parton(left) and a initial gluon jet parton (right) with different initial energy in a static and uniform medium with temperature $T=200 \mathrm{MeV}$ and $T=300 \mathrm{MeV}$.

path length $d E / d z$ of a quark (left) and a gluon (right) at different temperature. The decrease of the averaged parton energy loss per unit path length with the path length in a uniform medium is caused by the energy consumption of the leading parton via the multiple interaction as the theoretical estimation indicates [17]. This effect becomes stronger at higher temperature due to the larger scattering rate and interaction strength. The path length dependence for a gluon is much stronger compared to a quark because the gluon scattering rate is about 9/4 larger due to the different color factors in the calculation of the cross section.

In Fig. 2 we show the transverse momentum distributions of a quark and a gluon at different times. We can see a clear transverse momentum broadening as expected. We use a Gaussian fit as $\frac{d N}{d p_{T}}=\frac{2}{\left\langle p_{T}^{2}\right\rangle} p_{T} \exp \left(-\frac{p_{T}^{2}}{\left\langle p_{T}^{2}\right\rangle}\right)$, where $\left\langle p_{T}^{2}\right\rangle$ is the average transverse momentum square of the leading parton obtained from the simulation. We also find that the 

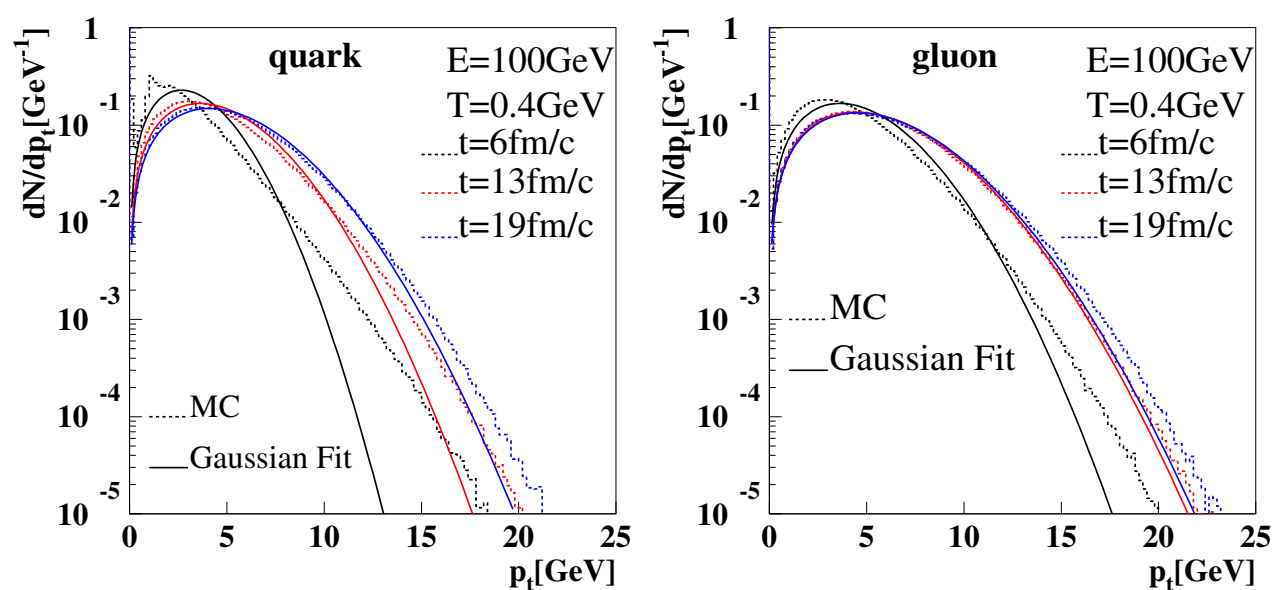

Figure 2. (Color online) Transverse momentum distribution (histogram) of the leading jet parton at different times. We consider a quark(left) and a gluon(right) jet parton propagating in a uniform and static medium at temperature $T=400 \mathrm{MeV}$. We also show Gaussian fits in the solid curves.

transverse momentum distribution of a gluon (right) becomes a Gaussian distribution at low $p_{T}$ region in later times, which takes longer for a quark (left) because of its smaller scattering rate. However, our simulation results show that the Gaussian fits fail at high $p_{T}$ region, which is consistent with the theoretical calculation [14], since such large transverse momentum of the leading parton is more likely to come from a rare single strong scattering than picking up the small transverse momentum transfer via multiple scatterings.

We now simulate the propagation of a gluon in a uniform and static medium to see how the interaction with the medium affects the energy and structure of the reconstructed leading jet. Shown in Fig. 3 is the leading jet energy

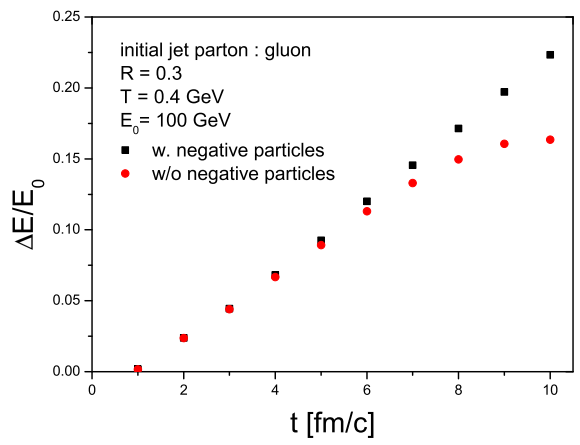

Figure 3. (Color online) The leading jet energy loss fraction in a static and homogeneous medium at temperature $T=400 \mathrm{MeV}$ as a function of path lenth(propagation time). The results that include all(leading+recoiled+negative) partons is presented in black symbols, the results without "negative" partons is presented in red symbols. We use a gluon with initial energy $E=100 \mathrm{GeV}$ as the initial jet parton and the cone size is set as $R=0.3$.

loss fraction as a function of path length (propagation time). One can see a linear dependence of the jet fractional energy loss on the path length. The energy loss is almost unchanged at the initial stage of the propagation when we include the "negative" partons since only few of them are produced, the energy loss is much larger at the later stage after the "negative" partons are included as the energy inside the jet cone is depleted by the jet-induced wake, which becomes significant as the jet propagates through the medium.

Shown in Fig. 4 (left) are the transverse profiles $\rho(r)=\frac{1}{\Delta r} \frac{1}{N^{\text {jet }}} \sum_{\text {jets }} \frac{p_{T}(r-\Delta r / 2, r+\Delta r / 2)}{p_{T}(0, R)}$ and (right) the longitudinal profile $d n / d\left(p_{L} / E^{\text {jet }}\right)$ (jet fragmentation function) of the leading jet at different times. As a consequence of the diffusion of the medium, a clear broadening of the leading jet transverse profile is observed. However, this broadening is 

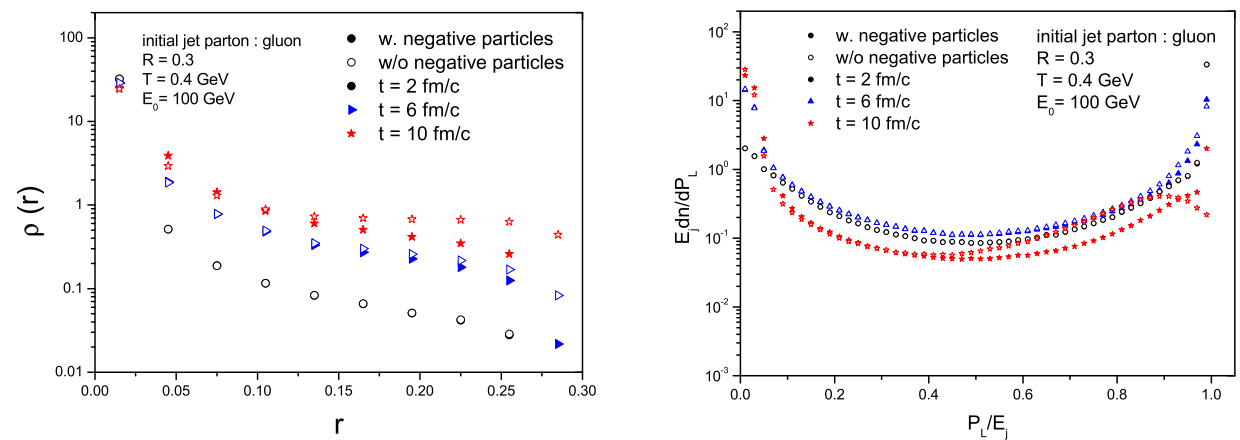

Figure 4. (Color online) (left) Transverse profiles and (right) longitudinal profiles of the leading jet at different times in a static medium at temperature $T=400 \mathrm{MeV}$. The results that include all(leading+recoiled+negative) partons are presented in solid symbols; the results without "negative" partons are presented in open symbols.

suppressed at later stage after the inclusion of the "negative" particles which are mostly distributed toward the outer annuluses of the jet cone. The enhancement at small $p_{L} / E^{\text {jet }}$ and the decrease at large $p_{L} / E^{\text {jet }}$ of the longitudinal profile shown in Fig. 4 (right) describe a picture that how the energy and momentum are redistributed inside the jet cone as the recoiled partons enter in and take the energy away from the leading parton. The inclusion of the soft "negative" partons leads to a shift to the small $p_{L} / E^{\text {jet }}$ area since the jet-induced wake will reduce the jet energy as shown in Fig. 3.

This work is supported by the NSFC under Grant No. 11221504, China MOST under Grant No. 2014DFG02050, the Major State Basic Research Development Program in China (No. 2014CB845404), U.S. DOE under Contract No. DE-AC02-05CH11231 and within the framework of the JET Collaboration.

\section{References}

[1] M. Gyulassy, I. Vitev, X. -N. Wang and B. -W. Zhang, Quark gluon plasma p123-191, ed. R. C. Hwa and X.-N. Wang [nucl-th/0302077].

[2] K. Adcox et al., Phys. Rev. Lett. 88, 022301 (2002).

[3] C. Adler et al., Phys. Rev. Lett. 89, 202301 (2002).

[4] S. Chatrchyan et al. [CMS Collaboration], Phys. Rev. C 84, 024906 (2011).

[5] K. Aamodt et al. [ ALICE Collaboration ], Phys. Lett. B696, 30-39 (2011).

[6] M. H. Thoma and M. Gyulassy, Nucl. Phys. B 351491 (1991).

[7] A. K. Dutt-Mazumder, J. e. Alam, P. Roy and B. Sinha, Phys. Rev. D 71, 094016 (2005).

[8] M. Djordjevic, Phys. Rev. C 74, 064907 (2006).

[9] G. Y. Qin, J. Ruppert, C. Gale, S. Jeon, G. D. Moore and M. G. Mustafa, Phys. Rev. Lett. 100, 072301 (2008).

[10] J. D. Bjorken, Fermilab-Pub-82/59-THY (1982) and erratum (unpublished).

[11] M. H. Thoma and M. Gyulassy, Nucl. Phys. B 351, 491 (1991).

[12] X. -N. Wang and Y. Zhu, Phys. Rev. Lett. 111, 062301 (2013).

[13] M. Cacciari, G. P. Salam and G. Soyez, Eur. Phys. J. C 72, 1896 (2012).

[14] F.D’Eramo, M.Lekaveckas, H.Liu, and K.Rajagopal, JHEP 1305 (2013) 031.

[15] H. Li, F. Liu, G. -L. Ma, X. -N. Wang and Y. Zhu, Phys. Rev. Lett. 106, 012301 (2011).

[16] J. Auvinen, K. J. Eskola and T. Renk, Phys. Rev. C 82 (2010) 024906.

[17] X.N. Wang, Phys. Rep. 280, 287 (1997). 